\title{
Induced Total Labellings of Models as Scale-free Networks
}

\author{
Bing Yao ${ }^{1}$, Jiajuan Zhang ${ }^{1}$, Xiangqian Zhou ${ }^{1}$, Xiang'en Chen ${ }^{1}$, Xiaomin Zhang ${ }^{1}$, \\ Ming $\mathrm{Yao}^{2}$, Mogang $\mathrm{Li}^{2}$ \\ ${ }^{1}$ College of Mathematics and Statistics, Northwest Normal University, Lanzhou, Gansu, China \\ ${ }^{2}$ Department of Information Process and Control Engineering, \\ Lanzhou Petrochemical College of Vocational Technology, Lanzhou, China \\ Email: yybb918@163.com, yybm918@163.com
}

Received April, 2013

\begin{abstract}
A recently discovered approach including de Brujin graphs and Eulerian circuits are used to DNA sequencing and fragment assembly, and to simplify DNA graphs through a series of transformations on graphs and digraphs in the field of bioinformatics. Since numbered graphs provide underlying mathematical models in studying the wide variety of seemingly unrelated practical applications, so graph colorings often are used to divide large systems into subsystems. A new graph labeling has been introduced and investigated.
\end{abstract}

Keywords: Bioinformatics; Network; Graph Labeling; Chromatic Numbers

\section{Introduction}

Scientists and researchers have emphasized DNA sequencing and fragment assembly with the hopes of enhancing their abilities to reconstruct full strands of DNA based on the pieces of data they are able to record in recent years. Complications arise with fragment assembly due to imperfect data sets. Strands are often riddled with repeats and come in varying sizes. As a result, configuring the image of the original genome is not as easy as fitting one puzzle piece into the next [7]. A recently discovered approach including de Brujin graphs and Eulerian circuits are used to DNA sequencing and fragment assembly, and to simplify DNA graphs through a series of transformations on graphs and digraphs in the field of bioinformatics [9].

In Operations Research or Systems Engineering Theory and Methods, one very often use graph colorings to divide large systems into subsystems. Coloring (di) graphs can be used to describe the street configuration [1], and distinguishing colorings [11] are related with traffic network and the frequency assignments of radio and satellites. Numbered graphs interpretations also apply to other areas of mathematics. Some of the most significant numerical results have result from the correspondence between some ruler problems in additive number theory and numbered graphs. Many applications of graph labeling can be found in [6].

Bloom and Golomb [2] have shown that numbered graphs provide underlying mathematical models in studying the wide variety of seemingly unrelated practical applications. For example, the design of certain important classes of good non-periodic codes for pulse radar and missile guidance is equivalent to numbering the complete graph in such a way that all the edge numbers are distinct; "nonnatural" methods of encoding the integers from 0 to $b^{n}-1$ using $n$-digit vectors from the b-symbol alphabet have been devised to minimize the seriousness of errors occurring in a single digit; determination of crystal structures from X-ray difference data has long been a concern of crystallographers; other applications of numbered graphs have included design of highly accurate optical gauging systems for use on automatic drilling machines, design of angular synchronization codes, design of optical component layouts for certain circuit-board geometries, and determining configurations of simple resistor networks which can be used to supply any of a specific set of resistance values. Bollobas and Pikhurko [4] introduce that a difference-magic labelling of a graph $G$ is an injective mapping $f: V(G) \rightarrow N$ (the set of all natural numbers) such that the labels $|f(x)-f(y)|$ of edges $x y \in E(G)$ are pair wise distinct. Clearly, every graph admits a difference-magic labeling, so a natural question to ask is how economical it can be. More precisely, the difference-magic number $\mathbf{D}(G)$ which is the smallest $k$ such that a difference-magic labeling of $G$ into $\{1,2, \ldots, k\}$ exists. And they also defined: A sum- magic labeling of a graph $G$ as an injection $f: V(G) \rightarrow N$ such that the labels $f(x)+f(y)$ of edges $x y \in E(G)$ are pair wise 
distinct. In fact, there are many edge labeling that are defined by $|f(x)-f(y)|$ or $f(x)+f(y)$ ([10], [6]).

New labellings. We are motivated from [4] and [6] to define two new labellings as follows. All elements of any number set mentioned are non-negative integers here. A $(p, q)$-graph is one on $p$ vertices and with $q$ edges. The symbol $[k, k+m]$ stands for the set $\{k, k+1, \ldots, k+m\}$, where integers $m>k \geq 0$. A set $S$ is called a $k$-set if it contains $k$ elements, i.e. $k=|S|$. The largest integer and the least integer in $S$ are denoted by $\max (S)$ and $\min (S)$, respectively. The graphs under consideration are simple and finite, undirected and loopless. We use the standard notation and technology of graph theory, or they can be found in [3] and [6]. We will show some connections between our labellings and well-known colorings, such as, the chromatic index $\chi^{\prime}(G)$ and the chromatic number $\chi(G)$ of a graph $G$.

A proper total coloring of a graph $G$ is defined on a subset of $V(G) \cup E(G)$ such that two adjacent or incident elements of the subset are colored with different colors. Similarly, a mapping $f$ from the vertex set $V(G)$ to $[0, q]$ is a proper vertex labelling of $G$ if $f(u) \neq f(v)$ for all edges $u v \in E(G)$. Write $f(V(G))=\{f(u): u \in V(G)\}$ and $f(E(G))=$ $\{f(u v): u v \in E(G)\}$, or $f(V)$ and $f(E)$ if there is no danger of confliction. Let $k=\max (f(V))$ and let $s$ indicate the number of distinct colors used in $f(V)$, we call $f$ a proper $(k, s)$-vertex labelling of $G$. Thereby, we can define two labellings as follows. Given a $(p, q)$-graph $G$ having a $(k, s)$-vertex labelling $f: V(G) \rightarrow[0, q]$. An induced difference edge-labelling $i_{f}^{-}$of $f$ in $G$ is defined as $i_{f}^{-}$ $(u v)=|f(u)-f(v)|$ for each edge $u v \in E(G)$; and an induced harmonious edge-labelling $i_{f}^{+}$of $f$ in $G$ is defined as $i_{f}^{+}$ $(u v)=f(u)+f(v)(\bmod q)$ for each edge $u v \in E(G)$.

Definition 1. A proper $(k, s)$-vertex labelling $f$ of a $(p, q)$-graph $G$, from $V(G)$ to $[0, q]$, is an induced difference total labelling (IDT labelling) of $G$ if its induced difference edge-labelling $i_{f}^{-}$is a proper edge coloring of $G$. We say $f$ to be a $(k, s, t)$-IDT labelling of $G$, where $t=$ $\left|i_{f}{ }^{-}(E)\right|$. The least number of $k$ spanning over all $(k, s, t)$-IDT labellings of $G$, denoted as $\chi^{-}(G)$, is called the IDT-hromatic number.

Definition 2. A proper $(k, s)$-vertex labelling $h$ of a $(p, q)$-graph $G$, from $V(G)$ to $[0, q]$, is an induced harmonious total labelling (IHT labelling) of $G$ if its induced harmonious edge-labelling $i_{h}^{+}$is a proper edge coloring of $G$. Let $t=\left|i^{+}{ }_{h}(E)\right|, f$ is also called a $(k, s, t)$-IHT labelling of $G$. The IHT-chromatic number of $G$, denoted as $\chi^{+}(G)$, is the smallest number of $k$ spanning over all $(k, s, t)$-IHT labellings of $G$.

An IDT labelling $f$ of a $(p, q)$-graph $G$ is consecutive if $\mathrm{f}(\mathrm{V})=[0, p-1]$. Analogously, an IHT labelling $h$ of $G$ is consecutive if $h(V)=[0, p-1]$. Clearly, every graph admits a $(k, s, t)$-IDT labelling and a $(k, s, t)$-IHT labelling. A $(k, s, t)$-IDT labelling of $G$ is strong if it satisfies two addi- tional requirements that $k=\chi^{-}(G)$ and $t$ is least, that is to say, for any $\left(k^{\prime}, s^{\prime}, t^{\prime}\right)$-IDT labelling of $G$ there is $t \neq t^{\prime}$. A consecutive $(k, s, t)$-IDT labelling of a graph $G$ may be a graceful labelling or a sequential labelling of a $(p, q)$-graph $G$ if $t=q$ (ref. [5], [6] and [13]), but a bandwidth labelling of $G$, such a counterexample is a star. A $(k, s, t)$-IHT labelling of $G$ may be a harmonious labelling or a felicitous labelling of $G$ if $t=q$ ([6], [12]).

Let $P_{4}=u v w x$ denote a path on 4 vertices. A consecutive $(3,4,3)$-IDT labelling $f$ of $P_{4}$ can be represented as $3 \underline{3} 022211$, where $f(u)=3, \quad i_{f}(u v)=\underline{3}, f(v)=0, \quad i_{f}(v w)=\underline{2}$, $f(w)=2, i_{f}(w x)=1, f(x)=1$. Again, $P_{4}$ has a $(3,3,3)$-IDT labelling $3 \underline{3} 0 \underline{1} 1 \underline{2} 3$ and a consecutive (3,3,2)-IDT labelling 2201123. We have that $\chi^{-}\left(P_{4}\right)=2$ since $P_{4}$ admits a strong (2,3,2)-IDT labelling $1 \underline{10} 2 \underline{2} 11$.

Definition 3. [14] Let $S$ be a nonnegative integer $k$-set containing zero. $S$ is called an ordinary antiaverage $k$-set if for any $a \in S$, the set $S$ satisfies $a_{i, 1}+a_{i, 2}+\ldots+a_{i, \beta} \neq \beta a$ for distinct $a_{i, j} \in S \backslash\{a\}, \quad 1 \leq j \leq \beta$. The ordinary antiaverage number, denoted by $\lambda^{(\beta)}(k)$, is equal to $\min _{S} \max (S)$ spanning over all ordinary antiaverage $k$-sets $S$.

It is easy to see that $\chi^{-}\left(K_{n}\right)=\lambda^{(2)}(n)$. We will use the following graphs.

(1) The join graph of two graphs $G$ and $H$, denoted by $G+H$, is defined by $V(G+H)=V(G) \cup V(H)$ and $E(G+H)=$ $E(G) \cup E(H) \cup\{u v: u \in V(G), v \in V(H)\}$.

(2) The kth power $G^{k}$ of $G$ is a simple graph with its vertex set $V\left(G^{k}\right)=V(G)$ and the edge set $E\left(G^{k}\right)=\{u v$ : $\left.\mathrm{d}_{G}(u, v) \leq k\right\}$, where $\mathrm{d}_{G}(u, v)$ indicates the distance between $u$ and $v$ in $G$. Obviously, $\Delta\left(G^{k}\right) \leq[\Delta(G)]^{k}$.

(3) A uniquely cycle graph $\mathrm{H}$ holds that the graph $\mathrm{H}-e$ will be a tree for any edge $e$ on a cycle of $H$.

(4) For each vertex $u$ of a connected $G$, its neighbor set $N(u)$ is $\left\{u_{1}, u_{2}, \ldots, u_{d}\right\}$, where $d=\mathrm{d}_{G}(u)$. We take another graph $H_{u, d}$ with vertex set $V\left(H_{u, d}\right)=\left\{v_{1}, v_{2}, \ldots, v_{d}\right\}$, and then delete the vertex $u$ from $G$, and next join $v_{i}$ to $u_{i}$ with an edge for $1 \leq i \leq d$. The resulting graph is denoted by $G\left(H_{u, d}\right)$, called a $H_{u, d}$-substitution graph of $G$. If each $H_{u, d}$ is a complete graph $K_{d}$, we write $G\left(K_{d}\right)$ instead of $G\left(H_{u, d}\right)$.

\section{Main Results}

Observation 1. Let $f$ be a $(k, s, t)$-IDT labelling of a graph G. (i) $\Delta(G) \leq \chi^{\prime}(G) \leq t \leq s \leq k$.

(ii) There is no path $P=u w v$ on 3 vertices such that $f(u)+f(v)=2 f(w)$.

(iii) The complementary labelling $g$ of the labelling $f$ is defined by $g(u)=k-f(u)$ for all $u \in V(G)$. Then $g$ is a ( $k, s, t)$-IDT labelling.

(iv) Any induced subgraph by two color classes in a $(k, s, t)$-IDT labelling of $G$ consists of isolated edges plus isolated vertices.

(v) If $f(u)=f(v)$, then $\mathrm{d}_{G}(u, v) \geq 3$. 
Observation 2. Let $G$ be a $(p, q)$-graph.

(i) $\chi^{-}(H) \leq \chi^{-}(G)$ for any subgraph $H$ of $G$,

(ii) $\chi\left(G^{2}\right) \leq \chi^{-}(G)$.

(iii) $p-1 \leq \chi^{-}(G) \leq \lambda^{(2)}(p)$ if the diameter of $G$ is not larger than 2 .

Lemma 1. If $f$ is an IDT labelling of a connected graph $G$, then $f$ is also an IHT labelling of $G$.

Lemma 1 shows that a graceful labeling of $G$ is also a harmonious labelling of $G$ [6].

Theorem 2. A connected graph $G$ holds

$\Delta(G) \leq \chi^{+}(G) \leq \chi^{-}(G)$.

Lemma 3. Let $K_{n}$ be a complete graph on $n$ vertices, $K_{m, n}$ be a complete bipartite graph on $n+m$ vertices; $W_{n}$ be a wheel, $F_{n}$ be a fan, $H_{n}$ be a helm, $P_{n}$ a path and $C_{n}$ a cycle on $n$ vertices, respectively

1) $\chi^{-}\left(K_{n}\right)=\lambda^{(2)}(n)$ and $n+3 \leq \chi^{-}\left(K_{n}\right)$ for $n \geq 5$.

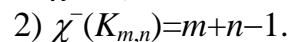

3) $\chi^{-}\left(W_{4}\right)=4, \chi^{-}\left(F_{5}\right)=5$ and $\chi^{-}\left(W_{n}\right)=n-1$ for $n \geq 6$.

4) $\chi^{-}\left(F_{n}\right)=n-1$.

5) $\chi^{-}\left(H_{2 n-1}\right)=n-1$ for $n \geq 8$, especially, $\chi^{-}\left(H_{2 n-1}\right)=7$ for $n=4,5,6,7$.

6) $\chi^{-}\left(P_{n}\right)=2$ for $n=3,4$, and $\chi^{-}\left(P_{n}\right)=3$ for $n \geq 5$.

7) $\chi^{-}\left(C_{n}\right)=4$ for $n=4,5$, and $\chi^{-}\left(C_{n}\right)=3$ for $n \geq 6$.

8) Cartesian product graphs $P_{m} \square P_{n}, \quad m \leq n$ hold $\chi^{-}\left(P_{2} \square P_{n}\right)=3, \chi^{-}\left(P_{m} \square P_{n}\right)=3$ for $m \geq 3$.

The number $\chi^{-}(G)$ can be referred to the partly antiaverage number for $f(u)+f(v) \neq 2 f(w)$ in $f(V(G))$. Since $\chi^{-}\left(F_{n}\right)=n-1$, however, the color set $f\left(V\left(F_{n}\right)\right)=[0, n-1]$ is not an antiaverage $n$-set at all. Another instance is that $\chi^{-}\left(K_{5}\right)=8$. It is easy to test $\chi^{-}\left(K_{5}-u v\right)=6$, where the graph $K_{5}-u v$ is obtained by deleting an edge $u v$ from $K_{5}$, using a coloring $f: V\left(K_{5}-u v\right) \rightarrow\{0,1,3,4,6\}$ such that $f(u)=6$ and $f(v)=3$. Therefore, we say that the color set $f(V(G))$ is partly antiaverage. Because diameters $D(G+H) \leq 2$ and $D\left(K_{m, n}\right)=2$, and any bipartite graph $G$ is a subgraph of a certain bipartite complete graph $K_{m, n}$. We have

Corollary 4. (i) For the join graph $G+H$ of two graphs $G$ and $H,|V(G)|+|V(H)|-1 \leq \chi^{-}(G+H) \leq \lambda^{(2)}(V(G)|+| V(H) \mid)$.

ii) Let $K(G)$ and $K_{s, t}$ be the order of the largest clique and a bipartite subgraph of a $(p, q)$-graph $G$ respectively. Then $\max \left\{\lambda^{(2)}(K(G)), s+t-1\right\} \leq \chi^{-}(G) \leq \lambda^{(2)}(p)$.

iii) For a bipartite graph $G, \chi^{-}(G) \leq|V(G)|-1$.

Theorem 5. Let $G$ be a $(p, q)$-graph. Then $\chi^{-}(G) \leq p-2$, otherwise $2 \sqrt{q} \leq \chi^{-}(G)$ or $2 \sqrt{q}-1 \leq \chi^{-}(G)$.

Theorem 6. Let $T$ be a tree. Then

$$
\Delta(T) \leq \chi^{-}(T) \leq \Delta(T)+1,
$$

and the bounds are tight. Furthermore, $T$ has a strong $(k, s, t)$-IDT labelling such that $t=\chi^{\prime}(T)$.

Theorem 7. A connected uniquely cycle graph $H$ holds $\Delta(H)+1 \leq \chi^{-}(H) \leq \Delta(H)+2$.

Corollary 8. Let $T$ be a tree with maximum degree $\Delta$, then $\chi^{-}\left(T\left(K_{d}\right)\right)=\lambda^{(2)}(\Delta+1)$, and $\chi^{-}\left(T\left(H_{u, d}\right)\right) \leq \lambda^{(2)}(\Delta+1)$.

For a subset $S$ of $V(G)$ whose cardinality $|S| \geq 2$, we say
$S$ a $k$-distance set of $G$ if the distance $\mathrm{d}_{G}(u, v)=k$ for any pair of two vertices $u, v \in S$. If mutually disjoint $k$-distance sets $V_{1}, V_{2}, \ldots, V_{n}$ of $G$ satisfies

$$
\sum_{i=1}^{n}\left(\left|V_{i}\right|-1\right) \geq \sum_{j=1}^{m}\left(\left|S_{j}\right|-1\right)
$$

for any group of disjoint $k$-distance sets $S_{1}, S_{2}, \ldots, S_{m}$ of $G$, thus, we can write

$$
\alpha_{k}(G)=\sum_{i=1}^{n}\left(\left|V_{i}\right|-1\right)
$$

and call this number the $k$-distance number of $G$, and $V_{1}$, $V_{2}, \ldots, V_{n}$ is a largest group of disjoint $k$-distance sets in $G$. It should be pointed out that there may be $n<m$ in (1), the cycle $C_{10}$ is such an example. In the following argument, we use $n_{k}(G)$ to indicate $n$ in $\alpha_{k}(G)$.

Observation 3. (i) Since a $k$-distance set $V_{i}$ satisfies $\left|V_{i}\right| \geq 2$, so $n_{k}(G) \leq|V(G)| / 2$.

(ii) Clearly, $\chi\left(G^{m}\right)=p-\alpha_{m+1}(G)$ for $m \geq 1$.

(iii) $\alpha_{k}(G)=0$ for $k \geq 3$ if and only if the diameter $D(G)$ $\leq 2$.

Theorem 9. Let $G$ be a connected $(p, q)$-graph with diameter $D(G) \geq 3$, then $\chi^{-}(G) \leq \lambda^{(2)}\left(\chi\left(G^{2}\right)\right)$.

Corollary 10. (i) Let $S_{i}$ be a 3-distance set of a connected $(p, q)$-graph $G$ for each $i \in[1, s]$. Le

$$
n=p-\sum_{i=1}^{s}\left|S_{i}\right|
$$

and $k=\max \left\{\left|S_{i}\right|: i \in[1, s]\right\}$, so $G \subset K(n ; s k)$ and then

$$
\chi^{-}(G) \leq \lambda^{(2)}(n+k) \text {. }
$$

ii) If $G$ is a connected $(p, q)$-graph with diameter $D$, and let

$$
n=p-\sum_{t=1}^{s}\lfloor(D-t+1) / 3\rfloor
$$

where $s=\lfloor D / 2\rfloor$, we have $\chi^{-}(\mathrm{G}) \leq \lambda^{(2)}(n+s)$. Coloring square graphs is related with the frequency assign problem, also is one of distance constrained labelings. Michael Molloy and M. R. Salavatipour (2005) announced: $\chi\left(G^{2}\right) \leq\lceil 5 \Delta(G) / 3\rceil+78$ for a planar graph $G$. In [8], Lih and Wang have shown: If $\Delta(G) \geq 3$ in an outerplanar graph $G$, then $\chi\left(G^{2}\right) \leq \Delta(G)+2$. If $\Delta(G) \geq 7$, then $\chi\left(G^{2}\right)=$ $\Delta(G)+1$. Thereby, we have

Corollary 11. Let $G$ be a planar and connected graph.

(i) Then $\chi^{-}(G) \leq \lambda^{(2)}(\lceil 5 \Delta(G) / 3\rceil+78)$.

(ii) If $G$ is outerplanar, $\chi^{-}(G) \leq \lambda^{(2)}(\Delta(G)+1)$ for $\Delta(G) \geq 7$, $\chi^{-}(G) \leq \lambda^{(2)}(\Delta(G)+2)$ otherwise.

\section{Conclusions and Future Works}

We wish applying $\chi^{-}(G)$ to approach some well-defined graph colorings, for example, $\chi\left(G^{2}\right) \leq \chi^{-}(\mathrm{G}) \leq \lambda^{(2)}\left(\chi\left(G^{2}\right)\right)$. We also define $\alpha_{n}(G)$ to set $\chi\left(G^{m}\right)=|G|-\alpha_{m+1}(G)$, but $\alpha_{n}(G)$ is not easy to be valued. In [14], it has been known that $\lambda^{(2)}(3)=3, \quad \lambda^{(2)}(4)=4, \quad \lambda^{(2)}(5)=8, \quad \lambda^{(2)}(6)=10, \quad \lambda^{(2)}(7)=12$, $\lambda^{(2)}(8)=13, \lambda^{(2)}(9)=19$ and $\lambda^{(2)}(10)=23$. It reveals to be not easy to gain the precise values of $\lambda^{(2)}(n)$. We propose 
Problem 1. Determine or seek some efficient bounds of $\lambda^{(2)}(n)$ for $n \geq 5$.

Conjecture 2. Let $G$ be a graph on $n$ vertices, there is $\chi^{-}(G) \leq 3(n-2)$.

Problem 3. Let integer $n \geq 5$. An IDT labelling $f$ of $K_{n}$ is optimal if max $f\left(V\left(K_{n}\right)\right)=\lambda^{(2)}(n)$ since $\chi^{-}\left(K_{n}\right)=\lambda^{(2)}(n)$. Let $C_{f}(u)=\{|f(u)-f(x)|: x \in N(u)\}$. Wether $C_{f}(u) \neq C_{f}(v)$ for all $u v \in E\left(K_{n}\right)$ under an optimal IDT labelling $f$ of $K_{n}$ ?

Our works show that some special classes of graphs which support Conjecture 4, such as trees, uniquely cycle graph, Halin graphs.

Conjecture 4. Let $G$ be a planar graph, then $\chi^{-}(G) \leq \Delta(G)+3$ and, $\chi^{-}(G) \leq \Delta(G)+2$ if girth $\mathrm{g}(G) \geq 5$.

Conjecture 5. For every cubic graph $G$, then $\Delta(G)+1 \leq$ $\chi^{-}(G) \leq \Delta(G)+3$.

Problem 6. We say a tree $T$ belongs to Class 1 if $\chi^{-}(T)=\Delta(T)$, otherwise it is in Class 2, i.e., $\chi^{-}(T)=\Delta(T)+1$. Characterize structures of trees in Class 1, or in Class 2.

It may be interesting to consider the averaged labelling of graphs. For a proper vertex coloring $f$ of a $(p, q)$-graph $G, f: V(G) \rightarrow[0, q-1]$, if a path $P=u w v$ of $G$ satisfies $f(u)+f(v)=2 f(w)$, we say $P$ an averaged path, and use the symbol $\lambda_{f}(G)$ to denote the number of all averaged paths in $G$. Find the maximum number $p_{\chi}(G)$ of $\lambda_{f}(G)$ spanning over all vertex colorings.

\section{Acknowledgements}

This research is supported by the National Natural Science Foundation of China in Grant No. 61163054 and Grant No. 61163037, and Research Projects of Graduate Teacher of Gansu University No. 1216-01. We thank Yuan Yao (Support Center of Cooperative Communication and Operation of China Telecom, Hangzhou, 310016, CHINA) for his useful suggestions of networks.

\section{REFERENCES}

[1] Jogen Bang-Jensen and Gregory Gutin, "Digraphs Theory, Algorithms and Applications,” Springer-Verlag. Berlin Heidelberg, New York, London, Paris, Tokyo, Hong Kong, Barcelona, Budapest, August 2007.
[2] G.S. Bloom and S.W. Golomb, “Applications of numbered undirected graphs,” Proc. IEEE, 65 (1977) 562-570. doi:10.1109/PROC.1977.10517

[3] J.A. Bondy and U.S.R. Murty, "Graph Theory with Application,” Macmillan, New York, 1976.

[4] Bela Bollobas and Oleg Pikhurko, "Integer Sets with Prescribed Pairwise Differences Being Distinct," preprinted

[5] Hui Cheng, Bing Yao, Xiang-en Chen and Zhong-fu Zhang, "On Graceful Generalized Spiders and Caterpillars,”. Ars Combinatoria 87 (2008), 181-191.

[6] Joseph A. Gallian, “A Dynamic Survey of Graph Labeling,” The electronic journal of combinatorics, 14 (2009), \# DS6.

[7] Jonathan Kaptcianos, “A Graph Theoretical Approach to DNA Fragment Assembly," American Journal Of Undergraduate Research, VOL. 7, NO. 1 (2008)

[8] Ko-Wei Lih and Wei-Fan Wang, "Coloring The Square of An Outerplanar Graph,” Taiwanese Journal of Mathematics, Vol. 10, No. 4 (2006), 1015-1023.

[9] Pevzner P.A., Tang H., and Waterman M.S, "A New Approach to Fragment Assembly in DNA Sequencing," $\begin{array}{llll}R E C O M B & 01 & \text { (2001), 256-267. }\end{array}$ doi:10.1145/369133.369230

[10] Alexander Rosa, "On certain valuations of the vertices of a graph,” Theory of Graphs (Internat. Symposium, Rome, July 1966), Gordon and Breach, N. Y. and Dunod Paris (1967), 349-355.

[11] J. Topp and P.D. Vestergaard, "\alpha_k\$- and \$ \gamma_k\$-stable graphs,” Discrete Mathematics 212 (2000), 149-160. doi:10.1016/S0012-365X(99)00216-2

[12] Yao Bing, Ming Yao, Hui Cheng, Jin-wen Li, Ji-guo Xie and Zhong-fu Zhang, "On Felicitous Labelling of Trees," Proceeding of The 4th International Workshop on Graph Labeling (IWOGL 2008), Harbin Engineering University and University of Ballarat, Australia, January, 2008, 5-8.

[13] Bing Yao, Hui Cheng, Ming Yao and Meimei Zhao, “A Note on Strongly Graceful Trees,” Ars Combinatoria 92 (2009), 155-169.

[14] Bing Yao, Ming Yao and Hui Cheng, "On Generalized Antiaverage Problem,” Ars Combinatoria Volume XCVI, July, 2010. 